\title{
OPEN-ENDED GENERALITY
}

\author{
Filippo Costantini
}

[This is a Post-print Manuscript (Author Accepted Version) of a paper appeared in The Philosophical Forum, June 2018, Wiley, pp. 161-191; DOI: 10.1111/phil.12182. Please quote from the final version: https://onlinelibrary.wiley.com/doi/abs/10.1111/phil.12182]

Abstract: In the debate on absolute generality, many authors have defended a relativistic position, namely that quantifiers are always restricted to a less than allinclusive domain. Consequently, they hold that an unrestricted quantification over everything is not possible. One problem for such a view is the need to explain the apparent absolute generality of logical laws, like $\alpha=\alpha$ or $\sim(\alpha \wedge \sim \alpha)$. The standard response appeals to schemas. In this paper, I begin by examining the reasons why schematic generality has such a strong appeal in this debate, which rely on their openendedness. However, I also raise an objection to show that schemas cannot be a good substitute for quantificational generality, due to the fact that they do not express propositions with a determined truth-value. The second part of the paper is dedicated to develop a different kind of generality, which is both open-ended (as schematic generality) and expresses a proposition with a determined truth-value (as quantificational generality). From a formal point of view, I will make use of a modal approach, in which the modality must be taken as primitive. The paper ends with a comparison of this form of generality and schematism, and argues that the former is to be preferred over the latter.

Key words: absolute generality, open-endedness, full schemas, modal approach, indefinite extensibility. 


\section{Introduction: the relativist position and the appeal of schemas}

One possible response to the set theoretic paradoxes is to deny that quantification over everything is possible. Notoriously, Russell was of this view, blaming impredicative definitions as the culprit of the paradoxes. His solution, the ramified type theory, bans impredicative definitions and does not permit a universal type, a type of all types. Ramified type theory is simple type theory with the supplementation of orders (sometimes called 'levels'). The universe is divided into infinitely many types: at type 0 there are individuals (things that are not classes); at type 1 there are classes of individuals (classes of type 0 object); at type 2 there are classes of classes of type 1, and so on. There is no universal type: each type has an immediately upper type. The type-stratification is not enough to ban impredicativity: for instance, at type 2 it is possible to quantify over all subclasses of type 1 (that is all subclasses determined by predicative or impredicative formulas). To avoid impredicativity, Russell adds 'orders', which introduce a stratification in the definitions of the classes. Apart from type 0 (where there are no classes), each type has infinitely many orders: at order 0 there are predicative classes (classes defined by means of individuals - if we are in type 1 - or by means of an inferior type for each type $>1$ ); at order 1 , there are classes defined by means of order 0 classes; at order 2, classes defined by means of order 1 classes, and so on. In this way impredicative definitions are prohibited: no class can be defined by means of the totality of classes to which it belongs.

In type theory, each quantifier is bound to a certain type, so that there cannot be a quantifier which ranges over all types. Nevertheless, we cannot avoid generalizing over any type: e.g. we may want to say that for each type there is an 
upper type. Russell and Whitehead proposed to consider generalization over types as typical ambiguous. A formula such as $\vdash \varphi(x)$ expresses a determined proposition with a truth-value only when we make explicit the type of its variables. Until then the expression is ambiguous, in the sense that different substitutions give rise to different propositions of different types. In contemporary terms, a typically ambiguous expression is a schema, not a sentence. For Russell and Whitehead, their idea was that in asserting a formula such as $\vdash \varphi(x)$ we are not asserting a single statement, rather we are asserting any of its instances ${ }^{1}$.

A similar use of schemas can also be found in the contemporary debate on absolute generality. Let's call 'absolutism' the position according to which an absolutely unrestricted quantification is possible. An absolutist is therefore someone who believes there to be an all-inclusive domain of objects and that this domain is available for us to quantify over. Let's call 'relativism' the position according to which no absolutely unrestricted quantification is possible. A relativist will argue either that an all-inclusive domain does not exist, or that, if such domain does exist, it is not available for us to quantify over².

\footnotetext{
${ }^{1}$ M. Potter, Wittgenstein's Notes on Logic (Oxford: Oxford University Press, 2008); pp. 196-197.

2 See A. Rayo and G. Uzquiano (eds.), Absolute Generality (Oxford: Oxford University Press, 2006); p. 2. Throughout the paper I take quantification to be classical quantification and its semantics to be classical semantics, which means that the determinacy of the truth-value of a quantified sentence requires the specification of a domain of objects to act as the universe of discourse. Here and in the paper I shall follow the standard use of the word 'domain', which does not indicate a set (or set-like collection) of objects, rather just the objects (or the plurality of objects, where the term 'plurality' is used as in plural logic).
} 
Relativism concerning quantifiers must deal with a prima facie counterexample to their position: the generality of logical laws. How can we express the generality of logical laws if no unrestricted quantification is available? In a similar way to Russell's and Whitehead's proposal, the general strategy relativists have used to address this problem has been to appeal to schemas. Schemas are used extensively in logic; as such it is not surprising that many authors have thought of them as possible substitutes for unrestricted quantification. If we cannot express the generality of logical laws by means of unrestricted quantification, because there is no authentic unrestricted quantification, then we can use schemas to express such generality. Or at least that is the basic idea.

The structure of the paper is as follows: in §2 I define schematic generality and demonstrate how it differs from quantificational generality; in $\S 3$, I introduce the notion of a 'full schema', which is meant to be a particular kind of schema that can substitute unrestricted quantification, before considering some of the reasons relativists have given for affirming that schematic generality is not reducible to quantificational generality. We will see that the fundamental characteristic of schemas is their openendedness ${ }^{3}$; §4 raises an objection against the use of schemas to express absolute generality, and shows that such a strategy suffers from some serious difficulties; §5 develops an alternative approach which does not suffer from such problems. This approach exploits modal notions with the aim of providing an open-ended form of generality; §6 compares the two approaches and defends the superiority of the modal

${ }^{3}$ See $\S 3.1$ for an explanation of this notion. 
account with respect to the schematic one; $§ 7$ concludes with a summary of the results of the paper.

\section{What is a schema?}

A schema is a system composed of a syntactic string of words or symbols, and placeholders (usually indicated by meta-variables, which are to be considered empty places), together with a side condition which explains how the placeholders (the metavariables) must be substituted to obtain some instantiations of the schema ${ }^{4}$.

Schemas are widely used in contemporary logic and mathematics, e.g. they are used to specify axioms and inference rules in a logical system or, more generally, to express logical laws as $A \vee \sim A$. The latter schema can be fulfilled in many ways, but the side condition prescribes us to substitute the propositional letter $A$ with a wellformed sentence of English and to take the two logical symbols as disjunction and classical negation, respectively. Moreover, they are also widely used to formalize theories, as first-order Peano Arithmetic $\left(\mathrm{PA}_{1}\right)$, which are not finitely axiomatizable 5 .

A schema is not a sentence of English (or, more generally, a sentence of a language), because it has some empty places (indicated by meta-variables), which must be fulfilled if we want to obtain a meaningful sentence. The meta-variables of a schema do not range over a domain of objects, rather they are placeholders to be substituted with the variables of the object language. Consequently a schema cannot

\footnotetext{
4 J. Corcoran and I. Samawi Hamid, "Schema", (2016) The Stanford Encyclopedia of Philosophy. https://plato.stanford.edu/archives/fall2016/entries/schema/.

${ }^{5}$ Another typical use of schemas can be found in semantics, when it comes to specify the conditions for the definiteness of truth. Tarski's definition of truth employs a schema, the famous T-schema.
} 
be true or false (it is not a truth-bearer): only its instantiations can be true or false. It is fundamental to keep in mind that a formula with free variables is not a schema. I shall follow Quine 6 in calling formulas with free variables 'matrices'. Once their variables are bound by quantifiers, matrices can occur as a part of statements, while this is not the case with schemas, which are just syntactic strings of symbols ("mere diagrams instrumental to the study of statements", as Quine (1945: 3) puts it).

Before proceeding, we will need to establish some definitions ${ }^{7}$ : a schema is closed if it does not contain any free variable (note that I am speaking of variables, not of the schematic meta-variables); a schema is a closure of a free schema if it is obtained by a free schema with free variables $x, y$ and $z$ by prefixing to it the universal quantifiers $\forall x, \forall y$ and $\forall z$. An instance of a schema is a statement obtained by substituting the schematic meta-variables of the schema. A closed schema is said to be valid if all its instances are true. Otherwise, it is invalid. A commitment to a schema is a commitment to the claim that the schema is valid.

Since a schema does not have any truth-value, it cannot, strictly speaking, ${ }^{8}$ be asserted. A commitment to a schema is not a commitment to the truth of the schema;

6 W. V. O. Quine, “On the Logic of Quantification”, (1945), Journal of Symbolic Logic 10, pp. 1-12.

7 These definitions are based on Quine's definitions in Quine (1945: 3).

8 S. Lavine, "Something About Everything: Universal Quantification in the Universal Sense of Universal Quantification" (2006), in A. Rayo and G. Uzquiano (2006: 98-148). Lavine (2006: 118) notes that even though schemas are not truth-bearers, they are not totally neutral with regard to truth: "Acceptance of a full schema is certainly not neutral with respect to truth: it commits us to truths, namely its instances, 
rather it is a commitment to its validity. We are committed to the instances of the schema. In other words, we are saying that all instances of the schema are true sentences. Notice that the statement 'all instances of the schema are true sentences' is naturally interpreted as a universally quantified sentence. Introducing the commitment to a schema in this way, we are introducing it by means of quantificational generality. From this point of view it seems that schematic generality cannot be considered a totally independent form of generality with regard to the quantificational one, precisely because it requires the latter to express the commitment to a schema. This means that this way of introducing schemas (which, by the way, is the standard way of introducing schemas) reduces them to quantificational generality. The notion of a full schema (to be introduced in §3) should avoid this reduction.

\subsection{Schemas and negation}

An important difference between schematic and quantificational generality concerns negation. A universally quantified sentence such as $\forall x P(x)$ is equivalent to the conjunction $P x_{1} \wedge P x_{2} \wedge P x_{3} \wedge \ldots$; its negation is $\sim\left(P x_{1} \wedge P x_{2} \wedge P x_{3} \wedge \ldots\right)$, which is $\sim P x_{1} \vee \sim P x_{2} \vee \sim P x_{3} \vee \ldots$. The scope of the negation is the whole sentence, and this is possible because the quantified sentence expresses a determined proposition with a truth-value. On the contrary, the negation of a schema $P S$ is equivalent to $\sim P x_{1} \wedge \sim$ $P x_{2} \wedge \sim P x_{3} \wedge \ldots$, the reason being that since the schema does not express a determined proposition, its negation is equivalent to the negation of each single instance of the schema.

and it blocks us from taking to be true sentences inconsistent with its instances [...]. I therefore take full schemas to be, in an extended sense, assertible" (emphasis added). 
If we want to use schemas as a substitution for quantificational generality, this might raise a problem ${ }^{9}$. How can we express schematically the negation of a quantified sentence? Consider the sentence 'no donkey talks': $\forall x(D x \rightarrow \sim T x)$. Its negation $\sim \forall x(D x \rightarrow \sim T x)$ means that there is at least one talking donkey. However, the negation of the schema $D s \rightarrow \sim T s$ is $\sim(D s \rightarrow \sim T s)$ : 'anything is a talking donkey' (Williamson (2003: 438), and Lavine (2006: 139)). In any case, it is easy to see how we can overcome this problem. The idea is to express the negation of a quantified sentence by means of a schema in an indirect way. If $\sim \forall x(D x \rightarrow \sim T x)$ is true, and so the schema $D s \rightarrow \sim T s$ is not valid, then we can add a new constant symbol $c$ so that the sentence ' $D c \wedge T c$ ' comes out true, and we can write the condition $(D s \rightarrow \sim T s) \vee$ $(D c \wedge T c)$ to capture the idea that either the schema is valid or it is not. Consequently, we can express that there is at least one talking donkey, but we cannot express it just by adding a negation in front of a schema. This method has been developed by Lavine: "for any full schema $\phi(s)$, we introduce a new constant symbol $c$ with axiom $\phi(s) \vee \sim$ $\phi(c)$ and use $\sim \phi(c)$ to serve as the negation of $\phi(s)$ " (Lavine (2006: 139)). This method seems to give the relativist the possibility of expressing negated quantified sentences by means of schemas. In addition, the relativist can exploit this to reply to another common objection against schemas: schemas are not apt to appear in the antecedent of a material conditional. The reason is that a material conditional $\alpha \rightarrow \beta$

\footnotetext{
9 Different authors - for instance, Williamson (in T. Williamson, "Everything" (2003), Philosophical Perspectives 17.1, pp. 415-465) and Priest (in G. Priest, Review of 'Absolute Generality', (2006) Notre Dame Philosophical Review, An Online Journal, http://ndpr.nd.edu/news/absolute-generality) - have considered this fact sufficient reason to reject the schematic approach. As I hope to make clear in the present paragraph, this conclusion is too hasty.
} 
is equivalent to $\sim \alpha \vee \beta$. If $\alpha$ is a quantified sentence and schemas cannot express the negation of a quantified sentence, then schemas cannot appear in the antecedent of a material conditional. But the method outlined above, offering a way of expressing the negation of a quantified sentence, shows that this objection fails. From this point of view, there seems to be no reason to think that schemas cannot express what quantification expresses.

\subsection{Two interpretations of schemas}

How do we interpret a schematic claim? In the literature, there are two different ways of interpreting schemas. The first is to interpret schemas as meaningless strings of symbols that can only give rise to meaningful sentences when their meta-variables are instantiated. For instance, Whitehead suggests this reading in a letter dated 27th January 1911 to Russell: "So far from that, my view is that our symbols remain mere unmeaning forms until the types of all the variables are determined". In a letter dated 29th January 1911, he added: "According to me until all ambiguities are definitely settled there is simply a sequence of meaningless shapes" (for the quotation of Whitehead's letter, see Potter (2008: 201)). Whitehead took this radical view because he was worried of making typical ambiguity expressions collapse into quantificational generality. His idea seems to be that if a typical ambiguous sentence expressed some meaning, then it would be very difficult to distinguish it from a standard quantified sentence over all types.

The second way of reading schematic generality is less radical. According to this view, schemas express a meaning, e.g. the schema $\alpha=\alpha$ expresses a certain meaning - the concept of being self-identical; however, because of the placeholders, 
a schema cannot express a determined proposition with a truth-value. The schema is a sort of indefinite claim that commits us not to a single truth, but to the truth of each of its instances.

The latter interpretation is certainly the most widespread one within the contemporary debate on absolute generality, and it is the interpretation we will presuppose in the next paragraphs. In fact, the former interpretation does not seem to provide a valid substitution of quantification generality. For instance, consider a general sentence such as 'Everything is self-identical'. This is certainly a meaningful sentence, which we can grasp. This means that if we read it as a schema according the first reading of schematic generality, our reading is completely inadequate to the task of translating such a sentence, because it would translate a meaningful sentence into a meaningless schema. In addition, as we shall see in more detail later, schemas have been used to express generality about a potential infinite ${ }^{10}$. But the problem with the first reading is, again, that schemas are not meaningful. Consequently, there would be no meaningful generalization over a potential infinite. The first reading is therefore completely unfit to be used as a substitution for quantificational generality, and for this reason we are going to focus our attention solely on the second interpretation.

However, one must notice that the second, less radical, interpretation of schemas (i.e. typical ambiguous sentence) is not available with the theory of types. The reason is simply that the theory of types required that we assigned a particular type to the meanings of each expression of the object language, and not only to the syntactic expression. Therefore schemas as $\alpha=\alpha$ cannot express a unique meaning;

10 See footnote 13 for a brief explanation of what I mean with 'potential infinite'. 
rather such an expression is assigned different meanings (different concepts) with different types. The second interpretation is possible for those who uses schemas with ontological hierarchies, i.e. people like Glanzberg or Lavine that argues for the non-existence of an absolute domain of quantification. They need schemas not because their language is typed (which in fact it is not), but because they believe there cannot be any unrestricted quantification over everything. Therefore, they can say that a schema has always the same meaning whatever domain we consider: no type restriction applies to them.

\section{Schemas as expressing absolute generality: full schemas}

\subsection{Full schemas and open-endedness}

As we have seen, the ordinary use in logic of schematic generality reduces it to quantificational generality. Of course, if schematic generality presupposes quantificational generality, then appealing to schemas cannot help the relativist in the absence of unrestricted quantification. However, some authors ${ }^{11}$ have defended the existence of a different kind of schema that should not be reduced to quantification. The most articulate defence is offered by Lavine (2006), who writes:

Fortunately, there is another form of generality more primitive than quantificational generality that will do the job: we can take the logical rules, for example, $\phi, \sim \phi \vdash \psi$, to be schemes used to declare that any instance is valid, where 'any' is to be sharply

\footnotetext{
11 This view has been defined by several authors. See for example M. Glanzberg, "Quantification and Realism", (2004) Philosophy and Phenomenological Research 69.3, pp. 541-572; G. Hellman, "Against 'absolutely everything”, (2006) in A. Rayo and G. Uzquiano (2006: 75-97); Lavine (2006) or C. Parsons, "The Problem of Absolute Generality", (2006) in A. Rayo and G. Uzquiano (2006: 203-219).
} 
distinguished from 'every': the statement of a rule, though it does involve generality, does not involve quantification. In our examples, $\phi$ and $\psi$ are [...] full schematic variables: 'full' is added to indicate that what counts as an acceptable substitution instance is open-ended and automatically expands as the language expands. (Lavine (2006: 118)).

A full schema is thus a schema not reducible to quantification (which implies that schematic generality is a form of generality independent from the quantificational one), on account of its open-ended nature.

What exactly does ‘open-endedness' mean? Following Glanzberg (2004), we can note that the truth of a quantified sentence such as $\forall x(x=x)$ does not depend on the domain of the quantifier: whatever domain we choose, that sentence will be true. This insensibility can be read as a form of domain-independence. On the contrary, the truth-value of a (standard) quantified sentence such as 'all the bottles are empty' $-\forall x(B x \rightarrow E x)$ - depends on the domain of the bound variable $x$ (the sentence 'all the bottles are empty' may be true, if the quantifier ranges over the bottles in my home; but it is certainly false, if it rages over all existing bottles). Standard quantified sentences like the latter require the specification of a domain for the quantifiers to range over, i.e. they require a universe of discourse that specifies the objects the quantifiers range over. Glanzberg concludes that, because of this difference, a sentence such as $\forall x(x=x)$ should be translated into the schema $\alpha=\alpha$. Full schemas are open-ended because they are domain-independent.

This difference is mirrored by the epistemological status of the two kinds of generalization. The domain-independency of the former implies that we do not need to look at how the world is to know that everything is self-identical, rather this is known to be case a priori. On the contrary, since the latter kind of generalization is domain- 
dependent, we must look at how the world is (less grandiosely: we must look at what the domain of quantification is) to know whether the sentence is true or false. Therefore, this kind of generality expresses a posteriori truths.

Lavine (2006) gives the most detailed defence of full schemas. The idea is the same: the validity of a schema does not depend on a domain of objects, but as the domain or the language expands, so too the substitution instances of their symbols expand. This is mirrored in a difference in the inferential role between full schemas and quantification. A universally quantified sentence is true when all elements of the domain satisfy the formula; this is not the case with a schematic generalization: even if the substitution of every element of the domain in the schema only gives rise to true instances, this is not enough to declare the schema valid, because it may happen that in a expansion of the domain, we will uncover some counterexample to it. Another example of a difference in the inferential role can be seen by considering the derivation of $S 0 \neq 0$ (with $S$ indicating the successor function on natural numbers) from $S n \neq 0$, where $n$ is a schematic letter. This inference can always be done; however, if $n$ were a quantificational variable, the inference would be valid just in case $n$ does not occur free in one of the premises of the argument (Lavine (2006: 120)).

A commitment to a standard schema such as $\alpha=\alpha$ is a specific commitment to a general sentence: 'all instances of the schema $\alpha=\alpha$ are true'. Full schemas are different: thanks to their open-endedness, they cannot be reduced to a quantificational generality. In relation to full schemas, we can only say that each single instance of the schema is true, but not that all of its instances are true. Borrowing a well-known expression of Wittgenstein, we can show that, given an arbitrary instance for the metavariables of the schema, the result of substituting the meta-variables with it gives rise 
to a true statement, but we cannot say that this is always the case. With an example: from the quantified sentence (whose intended domain is the set of natural numbers) $\forall x(\phi(x) \rightarrow \phi(S x))$ together with $\phi(0)$, we can infer $\forall x \phi(x)$; but from the schema $\phi(n) \rightarrow \phi(S n)$ and $\phi(0)$ we cannot infer the same general statement, because the schema does not make an assertion about all numbers, but merely provides a mechanism through which to make assertions about particular numbers (Lavine (2006: 121)). As Fine underlines ${ }^{12}$, the (full) schematic approach to absolute generality tries to split a general commitment to particular sentences (the instances), from a particular commitment to a general claim (the quantified sentence that should correspond to the schema).

\subsection{Full schemas for indefinitely extensible sequences}

Why is it not possible to derive the quantified sentence $\forall x(\phi(x) \rightarrow \phi(S x))$ from the schema $\phi(n) \rightarrow \phi(S n)$ ? In other words, from the schema $\phi(n) \rightarrow \phi(S n)$ why can we not derive the correspondent matrix (the formula with free variables) $\phi(x) \rightarrow \phi(S x)$ and then, by universal generalization, the quantified formula $\forall x(\phi(x) \rightarrow \phi(S x))$ ? This latter universally quantified sentence would be the general truth we are committed to, when we commit ourselves to the schema $\phi(n) \rightarrow \phi(S n)$. The reason relies on the fact that one must accept that we are working within an actual (infinite) domain in order for this reasoning to be valid. The quantified sentence requires the specification of a domain of objects, and if there are infinitely many objects, then the domain must be

12 K. Fine, "Relatively Unrestricted Quantification”, (2006) in A. Rayo and G. Uzquiano (eds.) (2006: 2044). 
an actual infinite. However, if we are working with a potential infinite (with an indefinitely extensible sequence $)^{13}$, we cannot translate the schema into a formula with free variables, precisely because the translation would bring us from an open-ended schema to a formula that requires a fixed domain of objects to be interpreted. The translation would just delete the central feature of full schemas: their open-endedness.

Thanks to their open-ended nature, full schemas are useful for expressing generality over a potential infinite sequence, e.g. an indefinitely extensible sequence. If a quantified sentence needs a domain to be interpreted, indefinite extensibility shows that it is always possible to find elements that do not belong to that domain. The domain can always be extended. Their open-endedness, which allows them not to be bound by some particular domain, makes schemas suitable for generalizing over an indefinitely extensible sequence.

One may suppose that if unrestricted quantification is possible and, consequently, a universal domain, which contains every entity, is available, there cannot be any real difference between quantificational and schematic generality. Lavine argues that things are different: if it is possible that something exists but it does not actually exist, then full schemas would express a commitment that we would have

13 Roughly speaking, a concept is indefinitely extensible if, for every definite totality of objects falling under it, it is always possible to find a more inclusive definite totality of such objects. It is clear that, to an indefinitely extensible concept, there corresponds an indefinitely extensible sequence of its extensions. This sequence can always be increased because, given an arbitrary extension of the concept, it is always possible to find a more inclusive extension of the same concept. In this sense, the sequence constitutes a potential infinite. Therefore, in what follows we shall use the expression ‘indefinite extensibility' and 'potential infinite' as synonyms. 
if it existed, while simple quantification cannot express this commitment. Being bound to a certain domain of object, a universal quantified sentence cannot express the commitment to objects that could have belonged to its domain, but do not in fact belong to it. To express this commitment in quantificational terms, it is possible to adopt a modal framework - for instance, by claiming that the sentence is necessarily true - but this shows that the full schemas are stronger than quantification generality ${ }^{14}$.

\section{3 'Any' and 'All'}

${ }^{14} \mathrm{~A}$ different way of arguing for the irreducibility of schematic to quantificational generality can be found in Hellman (2006), who proposes to consider the generality of a schema such as $\alpha=\alpha$ in a rather Carnapian way. According to such proposal, these kinds of sentences are analytical and a priori, because they just constitute stipulations regarding how words like 'object', 'thing', 'entity' are to be used in our language. According to Hellman, when we say $\alpha=\alpha$ we are actually saying "anything that we ever recognize as an entity at all will be assumed to obey this" (Hellman (2006: 95), emphasis added). So we are dealing here with a stipulation, which tells us how to use the words in the language. On the contrary, the quantificational generality has nothing to do with stipulation (by saying 'all swans are white', we are not stipulating that the swans are white!), so the two kinds of generality must be different. I do not find this argumentative strategy particularly persuasive. How can a logical truth like $\alpha=\alpha$ have a stipulative meaning? When some people stipulate something, as in a contract, they have simply arrived at an agreement, a common decision. Implicit in this is that there was the possibility of a different decision being taken (or of no decision being taken, as when no contract is signed). But, in the case at hand, there are no possibilities for arranging things in a different way: we cannot coherently say 'let's stipulate that $\alpha=\alpha$ is not valid'. For this reason, speaking of a stipulation concerning logical laws such as $\alpha=\alpha$ can only have a metaphorical meaning and, consequently, it is better to avoid such use. 
Another typical way of arguing for a generality that is different from the quantificational one makes appeal to the distinction between 'all' and 'any'. Here is Russell15:

Given a statement containing a variable $x$, say ' $x=x$ ', we may affirm that this holds in all instances, or we may affirm any one of the instances without deciding as to which instance we are affirming. The distinction is roughly the same as that between the general and particular enunciation in Euclid. The general tells us something about (say) all triangles, while the particular takes one triangle, and asserts the same thing of this one. But the triangle taken is any triangle, not some one special triangle; and thus although, throughout the proof, only one triangle is dealt with, yet the proof retains its generality.

Russell is here appealing to a typical way of proving general statements concerning objects of a particular kind: one has to consider an arbitrary object $o$ and prove that $P o$ is true. Since the object is arbitrary, the proof does not rely on any particular feature of the object, and therefore we are allowed to generalize the result and conclude that the general statement is true. In proving the result we do not consider all the objects of a certain kind, but only one arbitrary object. The idea is that the determiner 'any' exactly captures this 'arbitrary' generality. An immediate objection is that once it is proved that $P o$ is true, we can conclude that $\forall x P x$ is true. In this way, 'any' would not express a different form of generality. But the objection misses the point. Even if the latter conclusion is legitimate, it does not remove the difference

15 B. Russell, "Mathematical Logic as Based on the Theory of Types", (1908) American Journal of Mathematics, 30.3 pp. 222-262; §2. 
between a proof that proceeds by considering a particular but arbitrary case, and a proof that considers all the cases.

A more recent defense of the difference between 'all' and 'any' can be found in Dieveny ${ }^{16}$. However, we are not going to develop this point further, because it requires a deep linguistic analysis which exceeds the aims of this paper. Moreover, even in the case in which this analysis would in fact confirm that any-generality works differently from all-generality, this would not still be enough to show that schematic generality is irreducible to quantificational generality, the reason being that any-generality could be interpreted differently from schemas, as we suggest in §5. For this reason we prefer to put the problem of the difference between 'any' and 'all' to one side and concentrate on the difference we saw before regarding the open-endedness of full schemas. Therefore, in what follows we consider the authentic difference between universal quantification and schematic generality to rest upon the open-ended nature of the latter.

\subsection{The semantics of schemas}

Since schemas are not truth-bearers, when dealing with the semantics we are not directly dealing with truth, but rather with validity (which implies the truth of each instances of the schema). The problem consists exactly in expressing the validity of a schema. It is tempting to say that a schema is valid when all its instances are true, but

\footnotetext{
${ }^{16}$ See P. Dieveney, "Anything and Everything", (2013) Erkenntnis 78.1, pp. 119-140 and P. Dieveney, "Quantification and Metaphysical Discourse", (2014) Theoria 80.4, pp. 292-318.
} 
we already know that this cannot be the case with full schemas. What we should do is express it by means of a meta-linguistic schema:

One can, of course, formally specify the semantics of full schemes in a suitable metalanguage, but that isn't terribly helpful, since the metalanguage will also employ full schemes. Since the usual semantics for the quantifiers makes use of quantifiers in the metalanguage, I do not view the - fully parallel - situation for full schemes as in any way problematic. (Lavine (2006:119)).

Lavine is certainly right in claiming that the usual semantics for quantifiers employs the same quantifiers in the meta-language; in particular, meta-linguistic quantifiers are used to give the semantics of object language quantifiers, because quantifiers are considered primitive in a FO-language (at least one quantifier must be taken as primitive). The idea is that we grasp the truth-condition of the object language quantified sentence by means of the meta-language quantified sentence. Of course, this presupposes that we already have a previous grasp of the meaning of the quantifier in question ${ }^{17}$ : by means of this knowledge, the standard semantics manages to convey the meaning of an object language sentence. The case of schemas is fully parallel to the quantificational case. We should grasp how a schema works by means

\footnotetext{
${ }^{17}$ For our argument, one need not take a position on the debate concerning the meaning of quantifiers: perhaps the meaning of quantifiers is given by their inferential role in natural deduction, or maybe we can only grasp the inferential role because we already grasp what generality is; in any case, for our purposes what matters is that standard semantics works by presupposing a grasp of the meaning of quantifiers.
} 
of a meta-linguistic schema, and this is possible because schemas are meaningful and we certainly grasp what they express ${ }^{18}$.

The last paragraph is very important, insofar as it clarifies the relation between a full (valid) schema and its instances. We do not need to quantify over all instances of the schema to declare its validity, because - as just explained - the fact that schemas are meaningful guarantees that we can use them in stating their semantics. As happened in the case of negation that we treated in $\S 2.1$, rejecting schemas because they are standardly introduced by quantifying over all their instances proves to be a common but hasty conclusion to take.

\subsection{Some general remarks on full schemas}

In this section, we have seen a number of differences between full schemas and quantification. We have also seen that the differences in the inferential role depend on the fact that full schemas are open-ended, whereas quantification requires the specification of a fixed domain of objects to act as the universe of discourse.

The general picture that emerges from the considerations above is that the open-endedness of full schemas marks a true difference from quantificational generality. Since quantification requires the specification of the domain of discourse, it cannot be open-ended ${ }^{19}$. That is the reason why advocates of full schemas have

\footnotetext{
${ }_{18}$ Notice that this would not be possible with the first, more radical, interpretation of schemas: according to that interpretation, schemas are meaningless strings of symbols, and consequently there would be nothing to grasp.

${ }^{19}$ Of course, this must not be interpreted as if we were claiming that the inference rules for quantifiers were not open-ended. As a matter of fact, the inference rules for quantifiers ( $\forall$-introduction and $\forall$ -
} 
proposed using them for generality over a potential infinite (an indefinitely extensible sequence). We could say that schemas are open-ended because they express something that does not depend on the objects in any domain.

Their open-endedness means that full schemas are not reducible to quantificational generality. However, will this suffice to avoid the fact that a general commitment to particular truths implies a particular commitment to a general truth? That is the major question now posed. The need to avoid a particular commitment to a general truth stems from the fact that schemas are not truth-bearers. However, in the next paragraph, we will argue against this position. What we will see is that the open-endedness of schemas does not depend on their lack of truth-values, and as such, open-endedness is not sufficient to avoid a commitment to a general truth.

\section{An objection to the schematic approach to absolute generality}

\subsection{The objection}

The objection concerns the potential infinite. Consider sentences such as 'Some sets are not members of themselves' or 'Each ordinal has a successor', and suppose that both the concepts of set and ordinal number are indefinitely extensible.

elimination in Gentzen's natural deduction) do not determine the range of the quantifier (its extension). In first-order logic, this is clearly shown by the Löwenheim-Skolem theorem, which implies the existence of different models with different infinite sizes that make true exactly the same sentences of the languages. (See, for example, I. Einheuser, "The Model-Theoretic Argument Against Quantifying Over Everything", (2010) Dialectica 64.2, pp. 237-246). Even though the inference rules for quantifiers are open-ended (they are domain-independent), quantification generality is not open-ended because its semantics is not open-ended. 
Schematically interpreted, these sentences have a meaning, but not a truth-value. Above we have underlined that the authentic difference between schematic and quantification generality rests upon the open-endedness of the former. Since the latter requires the specification of a domain of individuals, it is bound to the specified domain. So we need schemas, which are open-ended, to express such general sentences. We already know Lavine's argument (paragraph 3.2 above) that the derivation of a quantified sentence such as ' $\forall x(\phi(x) \rightarrow \phi(S x))$ ' from a schema ' $\phi(n) \rightarrow \phi(S n)$ ' presupposes that we are working within an actual (infinite) domain. Lavine is arguing that if we work within a fixed universe, then a general commitment to particular truths implies a particular commitment to a general truth, because within a fixed domain, schematic generality would collapse into quantificational generality. In fact, as soon as we have fixed the domain, i.e. we do not consider the possibility of enlarging (or modifying) it, the open-endedness that characterizes schematic generality can no longer play any role. When we deal with a potential infinite, the openendedness allows schemas to generalize over each extension of the sequence. Lavine's position thus suggests that open-endedness ought to be charged with the impossibility of shifting from general commitments to particular truths to a particular commitment to a general truth.

Lavine's reasoning can be summed up as follows:

1) A general truth always presupposes a fixed domain of quantification;

2) Sentences such as 'Some sets are not members of themselves' or 'Each ordinal has a successor' do not have a fixed domain of quantification; 
3) Therefore, such sentences do not express general truths.

If Lavine is right in thinking that a general truth always requires the specification of a domain of objects, then generality about a potential infinite is only expressible by means of schemas. Notice that this amounts to taking the standard semantics for the quantifiers very seriously: when a fixed domain of objects for the quantifiers is not available, then there can be no general sentence with a determined truth-value. But no argument is given to support the idea that a general truth always requires the specification of a domain of objects. In other words, sentence 1, which is the key premise of the whole argument, is just assumed to be valid, not argued for. Moreover, this approach towards quantification is dubious: one could in fact argue by modus tollens that since there are general truths about potential infinite, the standard account of quantification is simply wrong. For instance, Van Inwagen ${ }^{20}$ took this view:

There are, I concede, philosophers who maintain that when one says 'Some sets are not members of themselves' or 'For every ordinal number there is a greater', what one says is meaningless unless in uttering these sentences one presupposes a domain of quantification - a particular set of sets, a particular set of ordinals. These philosophers are in the grip of a theory. They ought to reason by Modus tollens; they ought to reason that because it is true without qualification that there are sets that are not members of themselves and that for every ordinal there is a greater, that their theory about quantification is false.

${ }_{20}$ P. Van Inwagen, "Being, Existence and Ontological Commitment", (2009) in D. Chalmers, D. Manley and R. Wasserman (eds.), Metametaphysics. New essays on the Foundations of Ontology (Oxford: Oxford University Press, 2009: 472-506). 
Using Van Inwagen's expression, we may say that the defender of schematism is "in the grip of a theory": he considers the standard theory of quantification correct, and consequently it infers that some generalizations are illicit, while a supporter of Van Inwagen's line would take for granted that there are such generalizations and would conclude that the theory is wrong. I suspect that many people will agree with Van Inwagen's position, which seems more natural and more respectful of our linguistic practice. In any case, for what we have said both positions are legitimate.

However, some progress can be made by noticing that the presupposition for which a general truth always requires the specification of a domain of objects seems not only dubious, but simply mistaken. In fact there are cases of general truths that do not depend on the specification of a domain of quantification. These truths are domainindependent as schemas are, and consequently, they can be open-ended as schemas. I shall now provide two different examples of these truths. Their existence reveals that Lavine is wrong in thinking that the open-endedness is to be charged with the impossibility of going from a general commitment to particular truths to a commitment to a general truth. His mistake consists in taking for granted a feature of standard quantification theory. Of course, what these examples show is not that standard quantification is wrong, but rather that it only captures a certain kind of generality. In other words, they show the existence of a different kind of generality, which is both open-ended and expresses propositions with determined truth-values. The existence of such examples is interesting because it shows that it in principle possible to have a true generalization over a potential infinite, as we shall explain in $\S 5$. 
The first example consists of what Kant called analytic judgments, sentences such as 'all bachelors are unmarried' or 'all bodies are extended'. Such sentences are not sensitive to their single instances, because they express what we may call conceptual truths: they express propositions which are true just in virtue of the meanings of the words involved. We do not need to check every single bachelor to see whether or not he is married; we just need to know the definition of the word 'bachelor'. It is in virtue of the concept of bachelor that the previous sentence is true. Therefore, the truth-value of such generalizations does not depend on having previously specified a domain or a pool of candidates (a plurality of objects) as values for the quantified variable. No matter which pool of candidates we may consider, the truth-values of such sentences always remain the same.

That such sentences are not schemas but authentic general truths is shown by the fact that in uttering a sentence like 'all bachelors are unmarried', we utter something whose truth merely depends on how we have defined the concept of bachelor and being unmarried. In other words, we are in a position to exhibit the reason why such a sentence is to be regarded as true. It should be clear that we are committing ourselves to a general truth, and not to particular truths.

Other example sentences that do not depend on a previously specified domain are some necessary a posteriori truths such as 'all whales are mammals'. These kind of sentences are not a priori sentences, because it was an empirical discovery that whales are mammals, not fish: without looking at the world and at the animals that had been baptized ${ }^{21}$ as whales, it would not have been possible to know if the sentence

21 The word 'baptism' is here used in the technical sense of Kripke-Putnam theory of reference. 
'all whales are mammals' is true or false. But once the discovery was made, then the truth of the sentence is clearly not dependent on the domain of whales we may consider. If we discovered the existence of animals very similar to whales but which are not mammals, the normal reaction would be to say that those animals are not whales, and not that some whales are not mammals.

These two kinds of generalization are clearly domain-independent. This is not surprising, since both kinds of sentences concern necessary truths. Their domainindependence shows that we can have general truths about a potential infinite. Suppose that the concept of ordinal number is indefinitely extensible and, consequently, that the ordinals form a potential infinite. The sentence 'every ordinal has an immediate successor' presents the same structure as the sentence 'all bachelors are not married'. It is true in virtue of the way in which ordinals are usually defined in set theory that each ordinal has an immediate successor. It is an a priori truth that does not require us to check each ordinal case by case. The sentence is therefore domain-independent: it is true in any domain of any model of set theory. Here we clearly have a general truth concerning a potential infinite.

\subsubsection{Domain-independency and open-endedness}

Until now we have used the terms 'domain-independence' and 'openendedness' as interchangeable terms. However, for what follows it is useful to distinguish them in a more precise way. What I propose is to consider domainindependence as a broader phenomenon than open-endedness; specifically, openendedness is a particular case of domain-independence, i.e. the case in which no absolute domain for the bound variable is available. In this sense, open-endedness 
implies domain-independence but not vice versa. To clarify the point with an example, consider again the sentences 'All bachelors are unmarried' and 'Any ordinal has a successor'. In the first case, the truth of the sentence is domain-independent, as explained above. However, it is hardly open-ended, because there is no problem in supposing that there is a set of all bachelors. On the contrary, in the second case there cannot be any set of all ordinals: this is a case where domain-independence goes along with the impossibility of fixing an absolute domain of ordinals. My proposal is to reserve the term 'open-endedness' only for cases such as the latter.

\subsubsection{A possible misunderstanding of the examples}

Before proceeding we must deal with a possible misunderstanding of our two examples. In particular, one might complain that the first example 'all bachelors are unmarried' is just intensional logic, and consequently it only regards a relation between two concepts: the truth of that sentence simply means that the concept 'being unmarried' is part of the concept 'being a bachelor'. The fact that the truth-value of the sentence does not depend on any specific domain of objects does not mean that we have discovered a domain-independent form of quantification, but rather that we have completely gotten rid of quantification.

This worry is misplaced. First of all, we were not looking for a domainindependent form of quantification. As we explained above, standard quantification is not open-ended because of its semantics. What we were looking for was a different form of generality, which is both open-ended and expresses a proposition with a determined truth-value. And for sure an 'intensional' example such as 'all bachelors are unmarried' gives us what we need. Even if one regards such a sentence as 
expressing something concerning two concepts, it is undeniable that what it says is valid for any person recognized as a bachelor. By expressing a relation between two concepts, we are making a generalization about any bachelor. Secondly, friends of the schematic approach to absolute generality use schemas even in the case of general statements like 'Every ordinal has a successor'. But as we stressed above, this presents the same structure as the sentence 'all bachelors are unmarried'. Its truth only depends on how ordinals have been defined. In other words, such a sentence also expresses a relation between two concepts, the concept of being an ordinal and the concept of being a successor. Therefore, our use of intensional examples is pertinent because schemas are invoked to express generality in these cases too.

\subsubsection{The meaning of the examples}

It is clear that the schematist cannot try to reply to these two examples by arguing that what we take here to be general truths are in reality indefinite claims that do not express any proposition. The reason why this reply is not available is that the truth of such sentences does not depend on the specified objects of quantification, rather they depend on the definitions of the concepts involved (in the first case), and on the definitions along with the way in which reference works (in the second case). In both cases we know why they are true. If they were schemas, we would find ourselves in the awkward situation of having to explain not only why they seem to express true propositions, but also why it seems that we know why they are true. There is no doubt that whilst dealing with such sentences, we are dealing with authentic propositions with a determined truth-value. 
The existence of such generalizations casts doubt on the schematic approach for at least three reasons. First of all, their existence shows that the presupposition that a general truth requires the specification of a domain of objects is simply wrong: there are general true propositions which do not depend on any specific domain of quantification. Secondly, their existence shows that domain-independence and, in particular, open-endedness are not to be charged with the impossibility of shifting from a general commitment to particular truths to a particular commitment to a general truth. This means that open-endedness is compatible with the sentence expressing a true proposition. But as our exposition of the schematic approach has shown, openendedness was the key feature to argue for the split between a general commitment to particular truths and a particular commitment to a general truth. Therefore, the schematist has lost the main argument that she had in support of his view. Thirdly, the similarity of generalizations like 'each ordinal has an immediate successor' with 'all bachelors are not married' strongly suggests that the former expresses a true proposition like the latter.

These three points do not suggest that schematism is in itself incoherent, but they show that we have no reason at all to think that such generalizations are authentic schemas, i.e. indefinite claims. Since the most natural way of interpreting such generalizations considers them to be claims that express authentic propositions, it is in this way that we should interpret them. Of course, the open problem consists in understanding how to interpret the logical form of such general sentences; because of their open-endedness, they cannot be standard quantified sentences.

\section{A different proposal: expressing open-endedness by going modal}




\subsection{The need of a modal approach}

It should be clear that the two examples above show that those kinds of generalizations are domain-independent because concepts are domain-independent. Before we said that 'all bachelors are unmarried' expressed a domain-independent truth, and also that this truth relies only on the concepts involved. Therefore, its domain-independence relies on concepts, which must be thought of as domainindependent. The same could be said of the second example ('all whales are mammals'), even though in that case there was the additional element of reference. As a matter of fact, when you define a concept as 'being a bachelor' it does not matter the domain of bachelors you are actually considering. In $\S 4.2 .1$ we distinguished between domain-independence and open-endedness by proposing to interpret the latter as a particular case of the former. In particular, a domain-independent concept is open-ended when it is not possible to comprise in a domain (set or plurality) the totality of its instances. According to these definitions, a concept such as bachelor is domain-independent but not open-ended. Since open-endedness of (full) schemas was indicated as the main difference between quantificational and schematic generality, one may think that a sentence such as 'all bachelors are unmarried' can be formalized by means of standard quantification: $\forall x(B x \rightarrow \sim M x)$. No problems arise by considering the set of all bachelors and, consequently, standard semantics can be used to state the truth-conditions of such a sentence. Even if this might be granted, this way of proceeding hides the fact that the truth of the sentence does not depend on any domain we may consider in giving its semantics. Clearly such a formalization obscures the intensional nature of such a sentence. 
This intensional nature clearly appears when we consider concepts that are also open-ended. This is the case of indefinitely extensible concepts. Suppose again that the concept of ordinal number is indefinitely extensible. This means that it cannot be identified either with a particular extension (set) of ordinals or with all extensions of ordinals, simply because there is no such thing as all extensions. In other words, the inner intensional nature of concepts is in no way reducible to extensional entities. Once more, this cannot be read as the mere claim that a concept cannot be identified with the objects that actually instantiate it, because there could have been other objects that could have instantiated the concept, but that do not actually instantiate it. Indefinite extensibility implies that there cannot be something like the totality of all objects that can instantiate the concept. As a consequence, we must take concepts very seriously, as primitive and irreducible to their extensions.

We could specify the intuitive notion of concept with which we are working in the following way: a concept $P(x)$ is specified by means of some condition of application that tells us to what individual objects we can apply the concept. We shall express these conditions simply by means of a formula $\lambda x . \phi(x)$. For our present aims, it is important to focus on the fact that their central feature depends on being domainindependent. This means that a condition of application tells us that if some individuals satisfy the condition, then the concept applies to them. No reference is needed to a previous domain of quantification: whatever individual in any domain satisfies the condition falls under the concept ${ }^{22}$. Moreover, in this picture, concepts remain the

22 For instance, Linnebo (in Ø. Linnebo, "Sets, properties, and unrestricted quantification” (2006). In A. Rayo and G. Uzquiano (eds.) (2006); p. 157) defends a similar view concerning properties. 
same while their extension expands, which simply means that the same concept can be applied to new objects without being modified.

What we need is a logical way of expressing that a sentence is true in those cases where such open-ended concepts are in play. Clearly standard quantification won't do. One might appeal to schemas, but we already saw a number of difficulties of such an approach. Here I will propose a different way to go. The basic idea is to use the generality provided by the modal operator ' $\square$ ' (and its dual ' $\diamond$ ') to express a form of open-ended generality, independently of which domain we may consider ${ }^{23}$. A sentence like 'Every ordinal has a successor' would be formalized as follows (where $O(x)$ must be read as ' $x$ is an ordinal', while $S(x)$ as ' $x$ has a successor'): $\square \forall x(O(x) \rightarrow$ $S(x)$ ). The modal operator just expresses the fact that the truth of the quantified sentence within its scope does not depend on the values of the bound variable 24 . The

${ }^{23}$ In the literature there are different proposals that use modal logic to express a domain-independent form of generality. The most famous are Fine (2006) and Linnebo (in particular $\varnothing$. Linnebo, "Pluralities and Sets", (2010) Journal of Philosophy 107.3, pp.144-164 and Ø. Linnebo, "The potential hierarchy of sets", (2013) Review of Symbolic Logic, 6(2)). While Fine's proposal is only sketched, Linnebo's one is far more developed. I will exploit some of his technical work in a later section of the paper. However, our views profoundly differ on the philosophical justification of the modal operator. I explain the differences in footnote 26.

${ }^{24}$ An immediate objection would be the following: if we consider a domain of just $n$ ordinals (with $n$ a finite natural number), then the sentence 'each ordinal has a successor' expresses a false proposition, because the nth-ordinal has no successor in the domain. But this is not a problem for our view. For instance, if we just consider finite ordinals, the sentence 'each ordinal has a successor' is a theorem of PA. Since PA has an axiom that states that for each natural number there is an immediate successor (just apply the successor function), no model of PA can be based on a finite set (or plurality) of natural 
generalization will hold however you expand the starting domain of ordinals. I shall follow Linnebo (2010) in calling the combination of the modal operator and the quantifier 'modalized quantifier'.

To be more precise, suppose you have defined a first-order language with a standard semantics for it. In specifying the semantics, you have also specified a domain as the universe of discourse for the language. However, you believe in the existence of indefinitely extensible concepts, and consequently you believe that there cannot be any all-inclusive domain. How can you express that any domain can be increased? Or how can you express the idea that logical laws are valid no matter what domain you are considering? Standard quantification won't do. Consider the sentence: $\forall x \exists y(x \subseteq y)$ where $\mathrm{x}, \mathrm{y}$ are variables for domains. The sentence says that all domains are extensible. The problem is that this sentence is inconsistent, because the quantifiers require a universe of discourse which must comprehend all domains (since the sentence speaks about domains in general), and so this universe of discourse cannot be expanded into a more comprehensive domain, providing a counterexample to the same sentence. Here we can make appeal to the modal operator: $\square \forall x \diamond \exists y(x \subseteq$ $y$ ): no matter the domain $x$ you consider, it is possible to find a more comprehensive domain $y$. The same strategy can be used with logical law: for instance, that anything

numbers. On the contrary, if we also consider infinite ordinals, then the sentence 'each ordinal has an immediate successor' is a theorem of ZFC: no model of ZFC allows the existence of only finitely many individuals (or no model of ZFC can contain - let's say - only $\omega+17$ ordinals - another domain that would make the sentence false). It is therefore clear that when we take the sentence 'each ordinal has a successor' to be true, we take it to be true with regards to the models of a certain theory. 
is self-identical can be expressed by the following sentence: $\square \forall x(x=x)$. Again, the idea is that the domain of the quantifier $\forall x$ is indifferent.

Before characterizing the invoked modality from a logical point of view, we must be clearer about what the modality is and is not. Let us start by making explicit what this modality is not. In this regard, we must stress that the operator expresses neither a metaphysical nor a logical modality. If it expressed a metaphysical modality, then by saying that any domain can be extended, we would imply that this extension is something contingent, contrary to our intentions. For instance, we want to claim that the concept of set is indefinitely extensible, even though if sets exist, they exist by metaphysical necessity (at least this is the traditional view concerning pure sets, which we do not intend to challenge here). At the same time, it is not a logical modality. With 'logical modality' I mean the identification of possibility with consistency (something is possible if it is consistent), and necessity with 'being a theorem of a logical system' (something is logically necessary if it is a theorem of a logical system). If the modality were in this regard logical, then it would be totally superfluous, because we can express consistency and theoremhood without introducing modal operators. Moreover, it would not be easy to see why such a modality could help us with the problem of absolute generality.

In my view, the modal operator is only a formal tool to express the openendedness of concepts. Open-endedness is just domain-independence when there is no fixed totality of instances of a concept, which means that the operator expresses the primitive intensional nature of concepts. Of course, all concepts are intensional entities, and so are domain-independent; but as outlined above, most of them are compatible with the existence of a maximal domain of their instantiations. In such 
cases, we do not need the modal operator to generalize over them, because the existence of a maximal domain guarantees that standard quantification can do the work (even if it obscures the intensional nature of some generalizations). But when this maximal domain is not available, and the concept is open-ended, then we need the modal operator, because modal operators provide a form of cross-domain generality ${ }^{25}$. Using more traditional jargon, we could say that the operator expresses, from a formal point of view, the universality of concepts (where universality is, of course, to figure as open-ended $)^{26}$.

25 This point is explained in more detail in the next paragraph.

${ }^{26}$ This way of interpreting the modal operator is very different from that of both Fine and Linnebo. Fine connects the modality with a particular view in philosophy of mathematics, which he calls "procedural postulationism". In short, mathematics is founded on postulates which are not indicative in character, but they have an imperatival character, i.e. they are like instructions to perform a certain action, and thus they cannot properly be true or false (Fine (2005: 89-90)). For instance, in arithmetic the successor postulate is the postulate that tells us, for a given natural number, to introduce a single natural number that will be the successor of the given number. In this context the postulational possibilities $\diamond_{\alpha} A$ means that it is possible to execute the postulate $\alpha$ in such a way that $A$ is then true, while postulational necessity means that we can always execute the postulate. On the contrary, Linnebo grounded his account on a certain metaphysical interpretation of the iterative conception of set (see Linnebo (2010)), where sets are individuated in stages, and the elements of a set are prior to the set in a way that to individuate a set, we must already have individuated all its elements. This prior to relation is a primitive relation that grounds the primitive modal operator. In stage $n$, the existence of the set of all elements of stage $n$ is only potential, in the sense that if we individuate it (and we certainly can, because in stage $n$, all its elements are available), we expand the domain of stage $n$, and in this way we obtain a further stage in the hierarchy. Both these interpretations rely on some view in philosophy of mathematics, and as such are very specific. By contrast, my view relies on a certain view about concepts, and therefore 


\subsection{Semantics}

Standard semantics for modal languages makes use of Kripke's possible worlds framework. A model is defined as a triple $\langle W, R, I\rangle$ where $W$ is a set of worlds, $R$ an accessibility relations between worlds and $I$ an interpretational function that assigns different truth-values to different formulas of the language with regard to specific worlds (so a formula is said to be true in a world). The term 'world' must not be taken at face value as denoting a way the actual spatio-temporal world in which we live could be. This is only a possible interpretations of worlds, which - for present purposes - must be set aside. We regard worlds as sets of objects, i.e. mathematical objects. $W$ is therefore a set of sets of objects. The key feature that motivates our choice of a modal approach is the fact that formulas are evaluated in a world (which here means that formulas are evaluated with regard to a set of objects). A formula like $\square \varphi$ is true at a world $w_{1}$ if $\varphi$ is true in all worlds accessible from $w_{1}$. Therefore, in the world $w_{1}$ we can state a claim that also regards other worlds, which means that the claim expresses a sort of cross-domain generality. This is exactly the kind of generality we need with regard to indefinite extensibility. If there is no all-inclusive domain of ordinals, then every claim we state about ordinals is always in relation to a specific domain of ordinals that can be increased. Therefore, general open-ended claims such as 'Each ordinal has a successor' must always be evaluated in relation to a specific domain of ordinals, because there is no maximal totality of ordinals. The modal

is far more general. Notice that the concepts implicitly defined by the postulate in Fine's view and the concept of set in Linnebo's view turn out to be indefinitely extensible, and so open-ended. From this point of view, it seems that my view goes directly to the heart of the matter. 
approach thus allows us to express a truth within a specific domain of ordinals which remains valid however we expand the starting domain. Therefore, the modal approach gives us the two features of that kind of generality we were looking for: modal formulas express propositions with truth-value, and are open-ended (insofar as their truth does not depend solely on the domain in which they are evaluated).

In any case it should be clear that the standard semantics does not fit our aims. The problem is that it is based on set theory and to work out it requires the existence of a set $W$ of worlds. But such a set cannot exist when dealing with indefinitely extensible concepts. Since each extension of an indefinitely extensible concept would be treated as a world, the non-existence of a maximal extension means that there cannot be a set of all extensions/worlds. As a consequence, the semantics of a modal sentence such as $\square \varphi$ that we reported above is not available to us. That semantics quantifies over all the worlds accessible from the one in which we evaluate the sentence. But this is possible only if such worlds can be collected into a set.

The fact that we cannot state the semantics by quantifying over all extensions/worlds means that we cannot explain the meaning of the modal operator by means of quantification. This is not surprising since we are looking for an openended form of generality, and quantificational generality is not open-ended. What this implies is simply that the modal operator must be taken as primitive, i.e. it cannot be explained away by means of other notions when specifying its semantics. In other words, a sentence like $\square \forall x(x=x)$ cannot be interpreted as if we were quantifying over all objects in all domains, because there is no such thing as 'all domains'. 
The situation is similar to the one we saw in $§ 3.4$ concerning schemas. There we argued that the semantics of an object language full schematic generalization must be stated by means of a meta-language full schema. Here it is exactly the same. The semantics of an object language generalization that employs a modalized quantifier requires a meta-language generalization that employs a modalized quantifier. The reason is that we are dealing with a primitive notion that cannot be explained by means of other notions. If one would like to dismiss the whole account because of this circularity, then one should also dismiss the use of standard quantifiers, because standard quantifiers present the same circular pattern. For instance, consider the following sentence of a meta-language in which we state the truth-conditions of a firstorder universal sentence:

$$
V_{M, g}(\forall \alpha \phi)=1 \text { iff for every } u \in D, V_{M, g}(\phi)=1
$$

The universal sentence of the object language has been assigned a truth-value in the meta-language by means of an expression 'for every $u$ ' composed by a quantifier whose range is the domain of the model. What does 'for every $u$ ' mean? If you formalize it, the only possibility you have in first-order logic is to use the universal quantifier $\forall$ (or you can use an equivalent expression such as $\sim \exists \sim$ ). So we have explained the truth-conditions of the universal quantifiers by means of the same universal quantifier ${ }^{27}$. This circularity is inescapable: what it means is simply that a

${ }^{27}$ Of course, technically speaking the two quantifiers are not the same, since one belongs to the object language, the other to the meta-language. However, it is clear that they are of the same kind, which is what we meant by saying that we have explained the truth-conditions of the universal quantifiers by means of the same quantifier. 
quantifier (in first-order logic) must be taken as something primitive, not reducible to other entities.

The same must be said in relation to the way we specified the condition of application of concepts at the beginning of $\S 5.1$. In dealing with the condition of application of concepts, we said that a condition tells us that if some individuals satisfy the condition, then the concept applies to them. In stating the condition, we used a quantifier 'some' that must be taken as absolutely general. If so, it seems that the introduction of concepts is grounded on an absolutely general quantifier, which would make the operator we invoked totally useless. However, I think this impression is misleading. The expression 'some' is certainly a quantifier, i.e. an expression of generality, but there is no reason to interpret it according to standard logic. 'Some' is a natural language expression, and not a formalized one, which means that it is possible - at least in principle - to formalize it differently from how it is usually formalized. Indeed, what we are suggesting is that we should formalize it by means of a modalized quantifier. The fact that we used (and we are likely to use again) natural language expressions that are normally read as standard quantifiers to explain what is going on with the modal operator does not mean that we are reducing this form of generality to standard quantification. If one has the feeling that the explanation actually reduces the modality to quantification, this is just because the acquaintance with the standard theory of quantification is so rooted that one immediately interprets expressions of generality in natural language as if they always behave as standard quantification.

In any case, we can make explicit the impossibility of reading those expressions as standard quantifiers by slightly modifying our previous characterization: a condition 
of application for a concept $P$ tells us that, necessarily, if some individuals satisfy the condition, then the concept applies to them.

\subsection{Syntax}

In the previous paragraph we outlined the fact that the modal operator is primitive, and therefore cannot be explained by means of other - more basic resources. Primitive notions cannot properly be defined, but they can be characterized through their behavior. In the present case, this means that we must specify the logic that the operator follows ${ }^{28}$.

Let us start with standard first-order logic (FO) with the standard formation clauses for formulas and inference rules for connectives and quantifiers. I will adopt here an axiomatic approach. To FO-logic let us add the operator $\square$, to get modal predicative logic (MPL). Again, the formation clauses for modal formulas and the inference rules are standard. Which axioms regulate the behaviour of the operator? Of course, we need the K-axiom $(\square(\varphi \rightarrow \psi) \rightarrow(\square \varphi \rightarrow \square \psi))$, but what else? Recall that we need the modal operator to allow generality in the absence of an all-inclusive domain of discourse, i.e. when we deal with indefinitely extensible concepts. Therefore, we must look at the features of indefinite extensibility to establish the axioms we need. Indefinitely extensible concepts - like the concept of ordinal number

\footnotetext{
${ }^{28}$ Here I exploit some technical work done by Linnebo $(2010,2013)$ concerning his aim of formalizing ZF-set theory in a modal language. However, I have adapted his technical work for a different philosophical task: I am not interested here in giving a certain interpretation of the iterative conception of set (as Linnebo does); rather I am interested in characterizing a certain view of concepts as being open-ended.
} 
- form a linear order (the sequence of ordinal numbers, i.e. the sequence of the extensions of such a concept forms not only a linear order, but a well-ordering). A linear order is reflexive, antisymmetric, transitive and total (if $a$ and $b$ are two arbitrary elements and $R$ is the relation, then either $a R b$ or $b R a)$. By adding axioms for reflexivity $(\square \phi \rightarrow \phi)$ and transitivity ( $\square \phi \rightarrow \square \square \phi)$ we obtain the modal system S4. Concerning the antisymmetric property, for our aims it is enough not to add the $B$-axiom (that corresponds to the symmetric property). Do we have to also add the correspondent axiom for the total property? Instead of totality we prefer to add a weaker axiom that allows for the case in which a certain extension can be enlarged in different ways, provided that all these ways have a common upper extension that comprehends all of them. This corresponds to the so-called directedness property (G-Axiom: $\rangle_{\square} \phi \rightarrow$ $\phi)$. With this further axiom, we get the system $S 4.2^{29}$.

Earlier we said that indefinitely extensible concepts do not change while their extensions increase ${ }^{30}$. If $P$ is such a concept, then we can formally capture this feature by means of the following two conditions:

$$
\begin{gathered}
P(x) \rightarrow \square P(x) \\
\sim P(x) \rightarrow \square \sim P(x)
\end{gathered}
$$

29 This is the same system individuated by Linnebo (2013).

30 This is the case at least with the traditional way of considering indefinite extensibility, which we are following here. There is also a different way of interpreting indefinite extensibility known as the 'linguistic interpretation' which affirms that every time we expand an indefinitely extensible domain of - for instance ordinals - we reinterpret the word 'ordinal' such that it refers to more individuals. For such an interpretation, see G. Uzquiano, "Varieties of Indefinite Extensibility", (2015) Notre Dame Journal of Formal Logic 56 (1), pp. 147-166; I am not going to deal with such a view in the present context. 
Let us consider again the case of ordinals. If we regard an object $c$ as an ordinal, however we expand the domain of ordinals $c$ will remain an ordinal. The second condition says that if we recognize $c$ as not being an ordinal, however we expand the domain of ordinals $c$ will never be recognized as an ordinal. These two conditions together are known as stability conditions. Moreover, there is a further condition concerning extensions:

$$
U \leq U^{\prime} \rightarrow D(U) \subseteq D\left(U^{\prime}\right)
$$

This condition guarantees that nothing gets lost in the passage from one extension to a more comprehensive one $\left(U \leq U^{\prime}\right.$ should be read as $U$ has been expanded into $U^{\prime}$, while $D(U)$ must be read as the domain of $U$ ).

The two stability conditions are fundamental, especially if combined with the reflexivity axiom. If $\phi$ is a formula, then we have both $\square \phi \rightarrow \phi$ (reflexivity) and $\phi \rightarrow \square \phi$, which produces the collapse of the modality ${ }^{31}$. This feature alongside the fact that the underground logic is S4.2 makes available to us a theorem (proved by Linnebo (2013)) concerning the behavior of modalized quantifiers. Consider the theory $T^{\diamond}-$ the theory based on S4.2 and where every atomic predicate is stable (it respects the two conditions above). According to our definition of indefinite extensibility, every indefinite extensible concept is stable. Therefore, the theorem can only be read with regard to indefinitely extensible concepts. Let $\vdash_{T} \diamond$ be the relation of provability in $T C^{\diamond}$, and $\vdash_{T C}$ be the relation of provability restricted to the non-modal fragment of $T C^{\diamond}$, which is

\footnotetext{
31 See Linnebo (2013: 213-214) for a formal proof of the fact that in this setting $\phi, \diamond \phi$ and $\square \phi$ are logically equivalent. This result is exploited in the proof of the theorem.
} 
essentially FO. If $\phi$ is a formula, we call $\phi^{\diamond}$ the result of substituting every nonmodalized quantifier in $\phi$ with a modalized quantifier ( $\forall$ will be replaced by $\square \forall$, while $\exists$ will be replaced by $\diamond \exists$ ). A formula is fully modalized if and only if all its quantifiers are modalized. Then we have the following:

Theorem (Linnebo 2013): Let $\phi_{1}, \ldots, \phi_{n}$ and $\psi$ be non-modal formulas in S4.2., then we have

$$
\phi_{1}, \ldots, \phi_{n} \vdash_{T C} \psi \quad \text { iff } \quad \phi_{1}^{\diamond}, \ldots, \phi_{n}^{\diamond} \vdash_{T C} \diamond \psi^{\diamond}
$$

Proof: by induction on the length of formulas (see Linnebo 2013).

What the theorem guarantees is that the modalized quantifiers behave, from a proof-theoretic point of view, as the standard quantifiers. This is a positive result since they were introduced to allow generality in the absence of an all-inclusive domain, and therefore the theorem assures us that they depart from standard quantifiers just as much as we need to guarantee absolute generality. More specifically, this means that the modalized quantifiers respect all the laws of classical logic (of course provided that the basic non-modal theory respects the law of classical logic). They diverge from standard quantifiers only with regard to classical semantics. This clearly shows that indefinite extensibility challenges the way in which classical semantics has been developed and not the laws of classical logic. But the theorem also allows us to interpret standard quantifiers in many ordinary theories as implicitly modalized ${ }^{32}$.

\subsection{Some conclusive remarks on the modal approach}

32 For more details on the syntactic part I refer the reader to Linnebo (2013). 
In the previous paragraphs we have exposed some technical results developed by Linnebo within his potentialist interpretation of ZF-set theory. However, we have adapted his results to a rather different philosophical task, namely that of providing a suitable interpretation of a modality that should express the open-endedness of concepts. In the present context, the modal approach is not used to defend a certain view in philosophy of mathematics, but rather to develop a modal theory of concepts that takes into account the fact that some concepts are indefinitely extensible (and thus their extensions form a potential infinite). We adopted a standard way of understanding such concepts: by means of the individuation of new instances, the concepts do not change, while their extensions grow. However, one should note that we are not committed to the idea that all concepts behave in this way. It is enough that indefinitely extensible concepts do so. In fact, the modal operator is required only in the case of open-ended concepts. As explained above, in the other cases, standard quantification will suffice. It is at this precise point that the theorem above gains its importance. By showing that modalized quantifiers behave like standard quantifiers from a proof-theoretical point of view, the theorem guarantees the authentic difference between them in terms of semantics. And this is a fundamental result, because the reasons behind the introduction of the modal operator were precisely semantic (the request of a domain as universe of discourse is a key feature of semantics). Therefore, the theorem guarantees that the modal approach differs from the standard quantificational approach only inasmuch as it is needed to provide general claims in the absence of an all-inclusive domain. In a nutshell, the theorem clearly expresses that the only real difference between the two forms of generality consists in the openendedness of the modal approach. 


\section{Comparing the modal approach with schematism}

Both schematism and our proposal share the key feature of expressing an open-ended generality, which means that they can provide a form of cross-domain generality. Even in the absence of an absolutely unrestricted domain of quantification, we can express the unrestricted generality of logical laws or other intensional truths by appealing either to our modal approach or to schemas. However, the schema in itself is neither true nor false, not expressing any determined proposition. By contrast, the modalized quantifier expresses a generalization that has a truth-value: the truthvalues of a conceptual generalization depend on the concept involved or, in the case of the example 'all whales are mammals', it depends on both the concepts involved and how reference is supposed to work. In this regard, the modalized quantifier expresses a form of generality that, on the one hand, is reducible neither to standard quantification nor to schematic generality; while, on the other hand, it is characterized by the two fundamental aspects that pertain to quantification and schematic generality, respectively. As quantification, but differently from schematism, a modal quantified formula has a truth-value, and therefore it expresses a proposition with a determined truth-value; as a schema, but differently from quantification, a modalized quantifier is open-ended, that is to say, it does not depend on a particular domain of objects.

This is the fundamental difference between the two approaches. But this difference has important consequences on a number of other aspects. First of all, our approach avoids the awkwardness of being committed to the idea that what seem to be perfectly meaningful and true propositions - such as 'everything is self-identical' or 'every ordinal has a successor' - are in reality schemas, i.e. indefinite claims with no truth-value. Friends of schemas can say that this is the price to pay when confronted 
with the paradox. However, our approach interprets them as expressing perfectly meaningful and true propositions, exactly as they seem. In this regard, our approach seems preferable.

Secondly, the schematic approach is a real relativist approach to absolute generality. The schematist believes that there can be no true proposition about everything. Lavine concludes his defense of schematism by claiming:

Everything is not a communicable or learnable notion [...]. There is no affirmative reason to believe that everything is a coherent notion. Moreover, even metaphysicians can do without everything, and so there is just no need to introduce it as a mysterious but necessary idea. When we add that denying that there is such a thing as quantification over everything suggests the beginnings of an attractive approach to the paradoxes, we see that there is every reason to believe that there is no such thing as everything. (Lavine (2006: 146)).

There is no doubt that this is a relativist conclusion. However, there are numerous reasons to doubt whether things go as Lavine says, that is, that we can do without the notion of everything (see for example Williamson (2003: §§3-4) for many examples of situations where we can hardly cope without such a notion). In any case, in this regard our approach marks a significant departure from schematism, because our modal strategy allows us to have perfectly true propositions about everything, as $\square \forall x(x=x)$. The notion of 'everything' comes out as a perfectly meaningful notion in our account. Of course, it cannot be formally translated by means of a standard quantifier, but rather by means of a modalized quantifier. The modal approach is thus not a relativist approach; rather it should be interpreted as a non-standard absolutist 
approach. It is non-standard because it affirms that absolute generality is possible, despite the absence of an absolute domain of quantification.

Thirdly, our approach performs better than schematism even with regard to negation. Our non-standard absolutist approach seems to be closer to our standard linguistic practise concerning negated sentences than the schematic approach. In fact, in natural language, it is always possible to negate a sentence by prefixing to it the locution 'it is not the case that...'. To negate a modalized quantified sentence, it is enough to prefix a negation in front of it. In this respect, it exactly mirrors what happens in natural language. However, we already know that to express the negation of a general sentence by means of a schema, just prefixing the schema with a negation will not do, and that we need a more indirect method (see §2.1). The reason is precisely that a schema does not express a unique proposition, but just an indefinite claim. The behaviour of negation is a further suggestion that natural languages' generalizations - such as 'Each ordinal has a successor' - express unique propositions, and not indefinite claims, contrary to what the schematic approach says, and therefore it is another reason to prefer our approach.

In conclusion, there is another important point to underline. One might have the impression that our approach is far more complicated - by making appeal to a primitive modal operator - than the schematist approach. While the latter makes appeal to a standard logical device like schemas, the former invokes a 'strange' primitive modality. But there are at least two reasons why schematism is not better positioned than our approach in this regard. Firstly, schematism does not invoke standard schemas, but full schemas which are a specific way of interpreting schemas. Whoever accepts standard schemas is not forced to accept full schemas. Secondly, 
our modal approach should be looked at only as a formal way of capturing the idea that concepts are open-ended. What is now important to notice is that the openendedness of concepts seems to be something necessary for schematism too. Recall how semantics for full schemas works (see §3.4). We grasp how a schema works by means of a meta-linguistic schema, and this is possible because schemas are meaningful and we grasp what they express. For instance, a schema like $\alpha=\alpha$ expresses a meaning, which is presumably the self-identity concept. But since the schema is open-ended, the meaning it expresses is also open-ended. Consequently, schematism (at least the second less radical way of interpreting it - but we saw that the first more radical interpretation does not work) presupposes the open-endedness of concepts. From a philosophical point of view, a friend of schematism should be in a position to appreciate the core idea behind our approach. We might say that schematism and our approach are two different formal ways of developing the same philosophical thesis, i.e. that concepts are open-ended. However, I think that the previous points clearly speak in favour of the modal approach.

\section{Conclusion}

In this paper we have presented two different ways of developing an openended form of generality. The first part was dedicated to the exposition of schematism, a view to which many authors have appealed in the lack of unrestricted quantification. Together with friends of schemas, we have individuated open-endedness as the distinctive feature of schematic generality in comparison with quantificational generality. Quantificational generality is not adequate to deal with indefinite extensibility, i.e. the notion of a potential infinite, precisely because it is not openended. 
However, we have extensively criticized the schematic approach for a number of problems it presents. Essentially, these problems have a common root: schemas do not express a proposition with a determined truth-value. Moreover, $\S 4$ criticizes an argument that aims to motivate the use of schemas to express cross-domain generality. Our main critique focused on the first premise of that argument, which says that a general truth always presupposes a fixed domain of quantification. By means of intensional examples, we argued that the premise is false, because there are (intensional) generalizations that are domain-independent. Quantification thus expresses only a particular form of generalization. Our critique shows that openendedness is not to be charged with the impossibility of having a proposition with a determined truth-value. In this way our critique opens up a logical space in which to think of a different form of generality, which is both open-ended and a truth-bearer. §5 was dedicated to exposing some ideas about how to formally capture this kind of generalization. From a logical point of view, the basic idea consists in using a primitive modal operator to capture open-endedness, while from a philosophical point of view, the operator has been interpreted as a formal tool to express the open-endedness of (some) concepts.

The two approaches have been compared in $\S 6$ according to a number of different aspects, and it has been argued that there are good reasons to prefer the modal approach. In this regard, the most important difference lies in the fact that while schematism is a relativist position within the debate on absolute generality, the modal approach turns out to be a non-standard absolutist position. This is for sure a great result given the central role that absolute generality plays in our theorizing. 